\title{
Serum Reactive Oxygen Species Modulator I as a Novel Predictive Biomarker for Resected Lung Adenocarcinoma: A Retrospective Pilot Study
}

\author{
Boksoon Chang $\mathbb{D I}^{\prime}$ \\ In Kyung Hwang ${ }^{2}$ \\ Seung Hyeun Lee (D) \\ 'Division of Pulmonary, Allergy, and \\ Critical Care Medicine, Department of \\ Internal Medicine, Kyung Hee University \\ College of Medicine, Seoul, South Korea; \\ ${ }^{2}$ Department of Medicine, Graduate \\ School, Kyung Hee University, Seoul, \\ South Korea
}

Purpose: Reactive oxygen species modulator 1 (Romo1) is a key regulator of intracellular reactive oxygen species production. Previous studies have shown that Romol overexpression in tumor tissue is associated with poor clinical outcomes in various clinical settings for lung cancer treatment. The aim of the present study was to assess the predictive value of serum Romol in patients received curative resection for lung cancer.

Methods: Serum samples were collected from patients with lung adenocarcinoma who underwent surgical resection. Baseline serum Romol and carcinoembryonic antigen (CEA) levels before surgery were measured. Univariate and multivariate analyses were performed to identify whether serum Romol was associated with disease-free survival (DFS).

Results: A total of 77 samples were analyzed. Using the cut-off value of $866 \mathrm{pg} / \mathrm{mL}$, the population was classified into low $(\mathrm{n}=42,54.4 \%)$ and high $(\mathrm{n}=35,45.4 \%)$ Romol groups. The median DFS of the high Romol group was significantly shorter than that of the low Romol group (25.5 months vs not reached [NR], $p=0.0105)$. In addition, the median DFS of patients in the high CEA $(>2.9 \mathrm{ng} / \mathrm{mL})$ group was significantly shorter than those in the low CEA group (26.8 months vs NR, $p=0.0092)$. Multivariate analyses showed that both high Romol and CEA levels were independently associated with poor DFS (hazard ratio $[\mathrm{HR}]=2.19$; 95\% confidence interval $[\mathrm{CI}]: 1.14-8.37$, and $\mathrm{HR}=2.95 ; 95 \% \mathrm{CI}: 1.23-9.21$, respectively). Moreover, combination of these two biomarkers resulted in higher HR of 4.11 (95\% CI, 1.53-14.05) for DFS than those of Romo1 and CEA.

Conclusion: Elevated serum Romo1 levels were significantly associated with early recurrence in patients with lung adenocarcinoma treated with surgical resection. Serum Romo1 may be a promising predictive biomarker for this patient population.

Keywords: reactive oxygen species modulator 1, serum, biomarker, recurrence, surgery

\section{Introduction}

Lung cancer is the leading cause of cancer-related deaths worldwide. Globally, it accounted for 2.1 million new cases and 1.8 million deaths in $2018 .^{1}$ In Korea, approximately 27,000 new cases and 18,000 lung cancer-related deaths were reported in $2017 .^{2,3}$ Adenocarcinoma is the major histologic subtype constituting $60 \%$ of all cases of non-small cell lung cancer (NSCLC). ${ }^{4}$ Despite recent advances in new treatment modalities, including targeted therapy and immunotherapy, NSCLC still has a poor prognosis, with a 5-year survival rate of only $24 \%{ }^{5}$ Various serum proteins, such as carcinoembryonic antigen (CEA), cytokeratin 19 fragments, and squamous cell carcinoma antigen, have been studied and used as
Correspondence: Seung Hyeun Lee Division of Pulmonary, Allergy, and Critical Care Medicine, Department of Internal Medicine, Kyung Hee University College of Medicine, Kyungheedae-ro 23, Dongdaemun-gu, Seoul, 02447, South Korea

Tel +8229588511

Fax +8229681848

Emailv3mann@naver.com 
biomarkers to diagnose or predict the prognosis ofNSCLC. $^{6-9}$ However, they are not highly reliable for the prediction of clinical outcomes, which limits their utilization in clinical practice. Therefore, validated predictive biomarkers need to be identified for better management of patients with lung cancer.

Reactive oxygen species modulator 1 (Romo1) is a novel protein, identified for the first time in 2006. It is located in the mitochondrial membrane and functions as a key regulator of intracellular reactive oxygen species (ROS) production. ${ }^{10} \mathrm{~A}$ recent study revealed that Romo1 is a nonselective cation channel possessing characteristics similar to those of class II viroporins. ${ }^{11}$ Previous data have demonstrated that the expression of Romo1 is upregulated in various cancer cell lines, and Romo1-derived ROS plays a critical role in cancer progression and metastasis in various cancer cells. ${ }^{10,12,13}$ Moreover, Romo1 increases the invasive activity of both hepatocellular carcinoma (HCC) and breast cancer cells via the NF- $\mathrm{B}$ signaling pathway. ${ }^{14,15}$ In addition to having important role in cancer cell proliferation, Romol-derived ROS are associated with resistance to cytotoxic drugs. ${ }^{12,16}$ Cumulative evidence suggest that Romo1 expression has predictive and prognostic value in patients with cancer. Elevated Romol expression in tumor tissues was significantly associated with poor survival outcomes and high lymph node metastasis in patients with surgically resected colorectal cancer (CRC). ${ }^{17}$ Tumor Romol overexpression was significantly associated with poor survival and vascular invasion in patients with HCC who underwent curative resection. $^{18}$ In addition, high Romol expression was significantly associated with poor clinical outcomes in patients with NSCLC who underwent surgery or platinum-based chemotherapy. ${ }^{19,20}$ According to recent studies, Romo1 was associated with early recurrence or metastasis of lung cancer treated with radiotherapy. ${ }^{21,22}$ These results consistently support that Romol can be a promising predictive and prognostic biomarker in various treatment settings for malignancies. However, the clinical implications of serum Romol in patients with cancer are still unclear.

The aim of this study was to investigate the potential predictive value of Romol as a biomarker by examining the association between serum Romo 1 level, similar to tissue Romol expression, and clinical outcomes of patients with lung cancer who underwent surgery.

\section{Materials and Methods}

\section{Study Subjects and Data Collection}

We collected data of patients with lung adenocarcinoma who underwent curative surgical resection between May 2016 and December 2017 at Kyung Hee University Hospital, a tertiary referral hospital in South Korea. Patients who died within one month after surgery, patients with cancer of other type, and patients who received neoadjuvant chemotherapy or radiotherapy were excluded to reduce bias in the survival analysis. During the study period, 171 patients underwent surgery for lung cancer at our institute, including 90 patients with adenocarcinoma. Four cases were excluded because of insufficient number of samples, five were excluded due to unavailable survival data, and four were excluded according to the exclusion criteria described above. Finally, 77 patients were included in the analysis.

All patients underwent staging workup, including chest computed tomographic scan, brain magnetic resonance imaging, and ${ }^{18} \mathrm{~F}$-fluorodeoxyglucose positron emission tomography. Pathological staging was determined according to the 8th edition of the International Association for the Study of Lung Cancer TNM staging system. ${ }^{23}$ All stage IIA or higher patients and high-risk stage IB patients with poorly differentiated tumors, $>4 \mathrm{~cm}$ tumor size, vascular invasion, visceral pleural involvement, those who underwent wedge resection, or incomplete lymph node sampling received adjuvant chemotherapy according to the clinical guideline. Clinical data were obtained retrospectively by reviewing the medical records. The study protocol was approved by the Institutional Review Board of the Kyung Hee University Hospital (KHUH 2017-06003). Written informed consent was obtained from all study subjects. All studies were conducted in compliance with the Declaration of Helsinki.

\section{Sample Collection}

Serum samples were obtained from the Biobank of Kyung Hee University Hospital. According to the standardized protocol, peripheral blood $(10 \mathrm{~mL})$ was collected in $\mathrm{BD}$ Vacutainer $\mathrm{SST}^{\mathrm{TM}}$ tubes (BD Diagnostics, New Jersey, USA) from the subjects using phlebotomy procedures less than $24 \mathrm{~h}$ prior to the induction of anesthesia or administration of premedication, and the specimen was centrifuged at $1300 \times \mathrm{g}$ for $10 \mathrm{~min}$ at $4{ }^{\circ} \mathrm{C}$. The serum specimens were transferred to a fresh tube and stored at $-80^{\circ} \mathrm{C}$ until further use. 


\section{Measurement of Romol and CEA Levels}

Serum Romo1 and CEA levels were measured via enzyme-linked immunosorbent assay (ELISA), using a human Romol immunoassay kit (Wuhan EIAab Science Co., Ltd, Wuhan, China) and electrochemiluminescence immunoassay (ECLIA) using a human CEA kit (Roche Diagnostics, Germany), respectively, according to the manufacturer's instructions with some modifications. All samples were blinded to the lab technician who performed the assays. All levels were determined in duplicates.

\section{Statistical Analyses}

The cut-off for discriminating between low and high serum Romo1 and CEA levels was defined as the point with the lowest $p$ value for disease-free survival (DFS) by the Log rank test for all possible levels for each biomarker. The proportions of low and high levels of these biomarkers in different patient groups were analyzed using the Chi-square test or Fisher's exact test, as appropriate. Clinical outcomes were assessed using the DFS and overall survival (OS). DFS was defined as the period from the day of surgery to recurrence or death. OS was defined as the interval from the day of surgery to death from any cause. Data of patients without tumor recurrence or death were censored at the last follow-up. Correlations between survival outcomes and clinicopathological parameters were estimated by univariate analysis using the Log rank test, followed by Cox proportional hazard regression analysis. Parameters with $\mathrm{p}$ values $<0.1$ in the univariate analysis were included for the multivariate analysis. Kaplan-Meier method was used to estimate survival rates. $\mathrm{p}<0.05$ was considered as statistically significant. All analyses were performed using SPSS v.20.0 (IBM Corporation, Armonk, NY, USA).

\section{Results}

\section{Patients' Characteristics}

During the last follow-up, 32 patients showed recurrence, and 13 patients died. Forty-five patients had no recurrence and were alive, while 19 were alive with recurrent lung cancer. Among 32 patients with recurrence, one had a single lung nodule, two had metastatic mediastinal lymph node, three had brain metastasis, five had malignant pleural effusion, six had bone metastasis (spine and femur), seven had intra-abdominal metastasis (liver and adrenal gland), and eight had metastasis in two or more organs. One patient with a single lung metastasis underwent surgical resection followed by platinum-based chemotherapy. Patients with brain or bone metastases received palliative radiotherapy for the corresponding lesions, followed by systemic treatment. Among the recurrence cases, 11 were epidermal growth factor receptor $(E G F R)$-positive and treated with EGFR-tyrosine kinase inhibitors. The remaining 21 patients were treated with either platinumbased doublet $(n=18)$ or platinum-based doublet plus immunotherapy $(n=3)$. The median follow-up period of the population was 38.2 months (range, 3.3-66.0 months).

The clinical characteristics of the study population are summarized in Table 1. All subjects were from Korea, and their median age was 68 years (range, 45-82 years). Among them, 29 (37.7\%) patients were aged $\geq 70$ years, $46(59.7 \%)$ patients were female, and $67(87.0 \%)$ were non-smokers. Sixty-eight (88.3\%) patients had an Eastern Cooperative Oncology Group performance status (ECOG PS) of 0 or 1. Fifty-five (71.4\%) patients had stage I or II disease and $22(28.6 \%)$ had stage IIIA disease. Sixty-seven $(87.0 \%)$ patients had cancer with well or moderate differentiation. Thirty-five and 2 patients had EGFR-mutated and $A L K$-translocated cancer, respectively. Most patients in this population $(67 / 77,87.0 \%)$ underwent lobectomy.

\section{Association Between Levels of Romol and CEA, and Clinicopathological Parameters}

The median Romo1 and CEA levels of the overall study population were $516 \mathrm{pg} / \mathrm{mL}$ (range, 30-2,396) and $2.6 \mathrm{ng} /$ $\mathrm{mL}$ (range $0.5-5.5$ ), respectively. To evaluate which clinicopathological parameters were associated with Romo1 and CEA levels, we compared their median levels between groups for each parameter. The median Romol level was significantly higher in patients with advanced-stage disease than in those with early-stage disease ( $p=0.0459$, Table 1). In addition, the median CEA level was significantly high in patients with advanced disease $(p=0.0003)$ and poorly differentiated tumor $(p=0.0433$, Supplementary Table 1).

The optimal cut-off value for low and high Romo1 levels was determined to be $866 \mathrm{pg} / \mathrm{mL}$ by the Log rank test. Similarly, the cut-off for CEA levels was determined as $2.9 \mathrm{ng} / \mathrm{mL}$. Using these cut-offs, $42(54.6 \%)$ patients were allocated to the low Romo1 group, 35 (45.4\%) patients were allocated to the high Romol group, and 34 (44.2\%) and 43 (55.8\%) patients were allocated to the low 
Table I Serum Romol Levels According to Clinicopathological Parameters

\begin{tabular}{|c|c|c|c|}
\hline & $\begin{array}{c}\text { No. of } \\
\text { Patients (\%) }\end{array}$ & $\begin{array}{l}\text { Serum Romol } \\
\quad(\mathrm{pg} / \mathrm{mL})^{*}\end{array}$ & $p$-value \\
\hline All & $77(100)$ & $500(236-960)$ & \\
\hline \multicolumn{4}{|l|}{ Age } \\
\hline$<70$ & $48(62.3)$ & 508 (229-905) & 0.7335 \\
\hline$\geq 70$ & $29(37.7)$ & $488(240-962)$ & \\
\hline \multicolumn{4}{|l|}{ Sex } \\
\hline Male & $31(40.3)$ & $536(246-962)$ & 0.5800 \\
\hline Female & $46(59.7)$ & $469(216-960)$ & \\
\hline Smoking history & & & 0.6413 \\
\hline Never & $67(87.0)$ & $468(22 I-942)$ & \\
\hline Ever & $10(13.0)$ & 498 (209-884) & \\
\hline ECOG PS & & & 0.6847 \\
\hline 0.1 & $68(88.3)$ & $516(2|4-9| 1)$ & \\
\hline$\geq 2$ & $9(11.7)$ & $540(304-834)$ & \\
\hline Stage & & & 0.0459 \\
\hline I, II & $55(71.4)$ & $450(226-960)$ & \\
\hline IIIA & $22(28.6)$ & 744 (45I-987) & \\
\hline T stage & & & 0.7824 \\
\hline TI-2 & $67(87.0)$ & $536(246-940)$ & \\
\hline T3-4 & $10(13.0)$ & 549 (337-837) & \\
\hline $\mathrm{N}$ stage & & & 0.0944 \\
\hline No & $57(70.0)$ & $456(240-842)$ & \\
\hline$\geq \mathrm{NI}$ & $20(30.0)$ & $736(442-975)$ & \\
\hline \multicolumn{4}{|l|}{ Differentiation } \\
\hline Well/moderate & $67(87.0)$ & $520(246-972)$ & 0.1457 \\
\hline Poor & $10(13.0)$ & $275(210-550)$ & \\
\hline \multicolumn{4}{|l|}{ EGFR mutation $\dagger$} \\
\hline Negative & $40(53.3)$ & $446(216-802)$ & 0.1964 \\
\hline Positive & 35 (46.7) & $536(340-975)$ & \\
\hline \multicolumn{4}{|l|}{$A L K$ translocation $\ddagger$} \\
\hline Negative & $40(95.2)$ & $472(232-940)$ & 0.2593 \\
\hline Positive & $2(4.8)$ & 906 (850-962) & \\
\hline \multicolumn{4}{|l|}{ Surgical technique } \\
\hline Lobectomy & $67(87.0)$ & $464(226-940)$ & 0.1068 \\
\hline Segmentectomy & $10(13.0)$ & $843(802-1,078)$ & \\
\hline
\end{tabular}

Notes: *Presented as the median (interquartile range). †Analysis after excluding two ALK-positive patients. ‡Analysis after excluding 35 EGFR-positive patients. Abbreviations: Romol, reactive oxygen species modulator I; ECOG PS, Eastern Cooperative Oncology Group performance status; T, tumor; N, lymph node; EGFR, epidermal growth factor receptor; ALK, anaplastic lymphoma kinase.

and high CEA groups, respectively. We compared the distribution of patients according to the Romo1 and CEA groups. As shown in Table 2, high Romo1 was significantly associated with advanced stage $(p=0.0113)$, poorly differentiated cancer $(p=0.0369)$, and $E G F R$-wild type tumor $(p=0.0212)$. Similarly, high CEA level was significantly associated with advanced stage $(p=0.0006)$ and poorly differentiated tumor ( $p=0.0197$, Supplementary Table 2).

\section{Association Between Levels of Romol and CEA, and Clinical Outcomes}

Survival analysis results with respect to clinicopathological parameters are summarized in Table 3. Median DFS for all study subjects was 40.8 months (range, 8.2-58.5 months). In the univariate analysis, patients with advanced stage ( $p=0.0003)$ and $E G F R$-wildtype ( $p=0.0382)$ showed significantly shorter DFS. In addition, high Romo1 expression (25.5 vs not reached [NR], $p=0.0105)$ and high CEA (26.8 vs.NR, $p=0.0092$ ) were significantly associated with poor DFS. Multivariate analysis showed that advanced stage (hazard ratio $[\mathrm{HR}]=2.52,95 \%$ confidence interval $[\mathrm{CI}]$ : 1.09-5.86), high Romo1 level (HR $=2.19,95 \%$ CI: 1.14 8.37), and high CEA level (HR $=2.95,95 \%$ CI: $1.23-9.21$ ) were independently associated with poor DFS. Kaplan-Meier survival curves showed that patients with high Romol and high CEA levels were likely to have poor survival in terms of DFS (Figures 1A and B). To evaluate different predictive values of serum Romo1 according to different characteristics, we further analyzed and compared HRs stratified by clinicopathological parameters. As summarized in Supplementary Table 3, high Romo1 level was significantly associated with shorter DFS in the younger age $(\mathrm{HR}=4.25)$, female sex $(\mathrm{HR}=2.62)$, good ECOG PS $(\mathrm{HR}=2.70)$, early stage $(\mathrm{HR}=4.04)$, well-to-moderately differentiated tumor ( $\mathrm{HR}=2.85)$, EGFR-mutant tumor $(\mathrm{HR}=3.54)$, and lobectomy $(\mathrm{HR}=2.91)$ groups.

We then performed an exploratory analysis of OS. Due to the relatively short follow-up period, we compared the 4-year survival rate (4-YSR) instead of the median OS between groups. Survival analysis results for OS are summarized in Supplementary Table 4. The 4-YSR for all study subjects was $78.4 \%$. Univariate analysis showed that old age, advanced stage, and EGFR-wild type were significantly associated with poor OS (all $p<0.05$ ). In addition, high Romo1 and high CEA levels showed a nonsignificant trend of association with poor OS ( $p=0.0617$ and $p=0.0584$, respectively). Multivariate analysis showed that advanced stage (HR $=3.69,95 \%$ CI: $1.18-15.10)$ were independently associated with poor OS. High Romo1 $(\mathrm{HR}=2.47,95 \% \mathrm{CI}: 0.89-11.71)$ and high CEA 
Table 2 Distribution of Patients According to Different Serum Romol Levels

\begin{tabular}{|c|c|c|c|c|}
\hline & \multirow[t]{2}{*}{ No. of Patients (\%) } & \multicolumn{2}{|c|}{ Serum Romol (pg/mL) } & \multirow[t]{2}{*}{$p$-value } \\
\hline & & Low $(<866)$ & High $(\geq 866)$ & \\
\hline All & $77(100)$ & $42(54.6)$ & $35(45.4)$ & \\
\hline Age & & & & 0.1831 \\
\hline$<70$ & $48(62.3)$ & $29(60.4)$ & $19(39.6)$ & \\
\hline$\geq 70$ & $29(37.7)$ & $13(44.8)$ & $16(55.2)$ & \\
\hline Sex & & & & 0.1562 \\
\hline Male & $31(40.3)$ & $20(64.5)$ & $10(35.5)$ & \\
\hline Female & $46(59.7)$ & $22(47.8)$ & $24(52.2)$ & \\
\hline Smoking history & & & & 0.1268 \\
\hline Never & $67(87.0)$ & $39(58.2)$ & $28(58.2)$ & \\
\hline Ever & $10(13.0)$ & $3(30.0)$ & $7(70.0)$ & \\
\hline ECOG PS & & & & 0.5814 \\
\hline 0.1 & $68(88.3)$ & $36(52.9)$ & $32(47.1)$ & \\
\hline$\geq 2$ & $9(11.7)$ & $6(66.7)$ & $3(33.3)$ & \\
\hline Stage & & & & 0.0113 \\
\hline I, II & $55(7 \mid .4)$ & $35(63.6)$ & $20(36.4)$ & \\
\hline IIIA & $22(28.6)$ & $7(31.8)$ & $15(68.2)$ & \\
\hline T stage & & & & 0.1292 \\
\hline TI-2 & $67(87.0)$ & $35(49.2)$ & $32(50.8)$ & \\
\hline T3-4 & $10(13.0)$ & $7(70.0)$ & $3(30.0)$ & \\
\hline$N$ stage & & & & 0.8147 \\
\hline No & $57(70.0)$ & $32(56.1)$ & $25(43.9)$ & \\
\hline$\geq \mathrm{NI}$ & $20(30.0)$ & $10(50.0)$ & $10(50.0)$ & \\
\hline Differentiation & & & & 0.0369 \\
\hline Well/moderate & $67(87.0)$ & $40(59.7)$ & $27(40.3)$ & \\
\hline Poor & $10(13.0)$ & $2(20.0)$ & $8(80.0)$ & \\
\hline EGFR mutation* & & & & 0.0212 \\
\hline Negative & $40(53.3)$ & $9(33.3)$ & $18(66.7)$ & \\
\hline Positive & $35(46.7)$ & $22(62.9)$ & $13(37.1)$ & \\
\hline$A L K$ translocation $\dagger$ & & & & 0.4921 \\
\hline Negative & $40(95.2)$ & $30(48.4)$ & $32(51.6)$ & \\
\hline Positive & $2(4.8)$ & $2(100)$ & $0(0)$ & \\
\hline Surgical technique & & & & 0.3323 \\
\hline Lobectomy & $67(87.0)$ & $35(52.2)$ & $32(47.7)$ & \\
\hline Segmentectomy & $10(13.0)$ & $7(70.0)$ & $3(30.0)$ & \\
\hline
\end{tabular}

Notes: *Analysis after excluding two ALK-positive patients. †Analysis after excluding 35 EGFR-positive patients.

Abbreviations: Romol, reactive oxygen species modulator I; ECOG PS, Eastern Cooperative Oncology Group performance status; T, tumor; N, lymph node; EGFR, epidermal growth factor receptor; ALK, anaplastic lymphoma kinase.

$(\mathrm{HR}=2.39,95 \% \mathrm{CI}: 0.91-8.25)$ levels tended to be associated with short OS. Kaplan-Meier survival curves showed that patients with high Romol and high CEA levels were likely to have poor OS (Supplementary Figure 1).

\section{Combination of Two Biomarker for the Prediction of DFS}

Both high Romo1 and CEA levels were associated with poor DFS, and we further investigated the effectiveness of the combination of two biomarkers for the prediction of 
Table 3 DFS Analysis Results According to Clinicopathological Parameters of All Study Subjects

\begin{tabular}{|c|c|c|c|c|c|c|}
\hline & \multirow{2}{*}{$\begin{array}{l}\text { No. of } \\
\text { Patients (\%) }\end{array}$} & \multirow{2}{*}{$\begin{array}{l}\text { Median DFS } \\
\text { (Months) }\end{array}$} & \multicolumn{2}{|c|}{ Univariate } & \multicolumn{2}{|c|}{ Multivariate } \\
\hline & & & HR (95\% Cl) & $p$-value* & HR (95\% Cl) & $p$-value $f$ \\
\hline All & $77(100.0)$ & 40.8 & & & & \\
\hline Age & & & & 0.8614 & NA & \\
\hline$<70$ & $48(62.3)$ & 40.8 & Reference & & & \\
\hline$\geq 70$ & 29 (37.7) & 31.2 & $1.07(0.52-2.18)$ & & & \\
\hline Sex & & & & 0.0992 & & 0.1817 \\
\hline Male & $31(40.3)$ & 46.4 & Reference & & Reference & \\
\hline Female & $46(59.7)$ & 28.7 & $1.92(0.89-4.15)$ & & I.87 (0.75-4.67) & \\
\hline Smoking history & & & & 0.2568 & NA & \\
\hline Never & $67(87.0)$ & 45.1 & Reference & & & \\
\hline Ever & $10(13.0)$ & 35.8 & $\mathrm{I} .7(0.69-4.5 \mathrm{I})$ & & & \\
\hline ECOG PS & & & & 0.4789 & NA & \\
\hline 0.1 & $68(88.3)$ & 41.8 & Reference & & & \\
\hline$\geq 2$ & $9(11.7)$ & 36.1 & $1.2(0.4 I-3.25)$ & & & \\
\hline Stage & & & & 0.0003 & & 0.0312 \\
\hline I, II & 55 (7I.4) & NR & Reference & & Reference & \\
\hline IIIA & $22(28.6)$ & 20.7 & $3.68(1.80-7.52)$ & & $2.52(1.09-5.86)$ & \\
\hline T stage & & & & 0.3340 & NA & \\
\hline TI-2 & $67(87.0)$ & NR & Reference & & & \\
\hline T3-4 & $10(13.0)$ & 40.8 & $1.65(0.60-4.58)$ & & & \\
\hline $\mathrm{N}$ stage & & & & 0.0817 & & 0.2770 \\
\hline No & $57(70.0)$ & NR & Reference & & Reference & \\
\hline$\geq \mathrm{NI}$ & $20(30.0)$ & 26.8 & $2.18(0.91-5.25)$ & & $0.50(0.14-1.75)$ & \\
\hline Differentiation & & & & 0.2690 & NA & \\
\hline Well/moderate & $67(87.0)$ & NR & Reference & & & \\
\hline Poor & $10(13.0)$ & 40.8 & $1.66(0.68-4.04)$ & & & \\
\hline EGFR mutation $\dagger$ & & & & 0.0382 & & 0.8104 \\
\hline Negative & $40(53.3)$ & 25.5 & $\mathrm{I} .88(\mathrm{I} .0 \mathrm{I}-5.2 \mathrm{I})$ & & $1.12(0.45-2.76)$ & \\
\hline Positive & $35(46.7)$ & NR & Reference & & Reference & \\
\hline Surgical technique & & & & 0.2371 & NA & \\
\hline Lobectomy & $67(87.0)$ & 46.4 & Reference & & & \\
\hline Segmentectomy & $10(13.0)$ & 36.9 & $1.8(0.68-4.75)$ & & & \\
\hline Romol level & & & & 0.0105 & & 0.0305 \\
\hline Low & $42(54.6)$ & $N R$ & Reference & & Reference & \\
\hline High & $35(45.4)$ & 25.5 & $2.30(1.21-5.98)$ & & $2.19(1.14-8.37)$ & \\
\hline CEA level & & & & 0.0092 & & 0.0320 \\
\hline Low & $34(44.2)$ & NR & Reference & & Reference & \\
\hline High & $43(55.8)$ & 26.8 & $2.91(1.30-6.50)$ & & $2.95(1.23-9.21)$ & \\
\hline Risk group & & & & 0.0017 & & 0.0079 \\
\hline Low & $27(35.1)$ & NR & Reference & & Reference & \\
\hline High & $50(64.9)$ & 21.3 & $3.34(1.57-7.09)$ & & $4.11(1.53-14.05)$ & \\
\hline
\end{tabular}

Notes: *Univariate analysis $\mathrm{p}$-value by Log rank test. †Multivariate analysis $\mathrm{p}$-value by Cox proportional hazard regression.

Abbreviations: HR, hazard ratio; Cl, confidence interval; ECOG PS, Eastern Cooperative Oncology Group performance status; T, tumor; N, lymph node; EGFR, epidermal growth factor receptor; ALK, anaplastic lymphoma kinase; Romol, reactive oxygen species modulator I; CEA, carcinoembryonic antigen; NA, not applicable. 
DFS. We divided our study population into two groups: the high-risk group, which included patients with high levels of both biomarkers simultaneously; and the lowrisk group, which was defined by the population other than the high-risk group. As shown in Table 3, 27 $(35.1 \%)$ and $50(64.9 \%)$ patients were allocated respectively to the two groups. Univariate analysis showed that the median DFS was significantly shorter in the high-risk group than in the low-risk group ( 21.3 vs $\mathrm{NR}, p=0.0017$ ). Multivariate analysis showed that the high-risk group was independently associated with poor DFS ( $\mathrm{HR}=4.11,95 \%$ CI: 1.53-14.05). Kaplan-Meier survival curves showed that patients in the high-risk group were likely to have poor DFS (Figure 1C).

\section{Discussion}

Identifying high-risk patients after surgical resection is critical because their survival can be prolonged by using adjuvant treatment. However, till date, there is no biomarker that has been widely accepted and clinically utilized for the prediction of high risk of recurrence after lung cancer surgery. The present study demonstrated that elevated serum Romo1 level was associated with early recurrence of surgically resected lung adenocarcinoma. Based on the HR, the discriminative power of Romo1 to predict recurrence after surgery was similar to that of CEA. To the best of our knowledge, this is the first study demonstrating that serum Romol level is associated with clinical outcome and has potential as an adverse predictive marker in patients with cancer. Our data suggest that patients with high Romol at baseline have high risk of recurrence, which may require a more aggressive treatment approach.

Romol is a novel protein that was first identified in a patient with head and neck cancer who was resistant to chemotherapy after recurrence. ${ }^{24}$ Studies have revealed that Romo1 is a major regulator of intracellular ROS

\section{A}

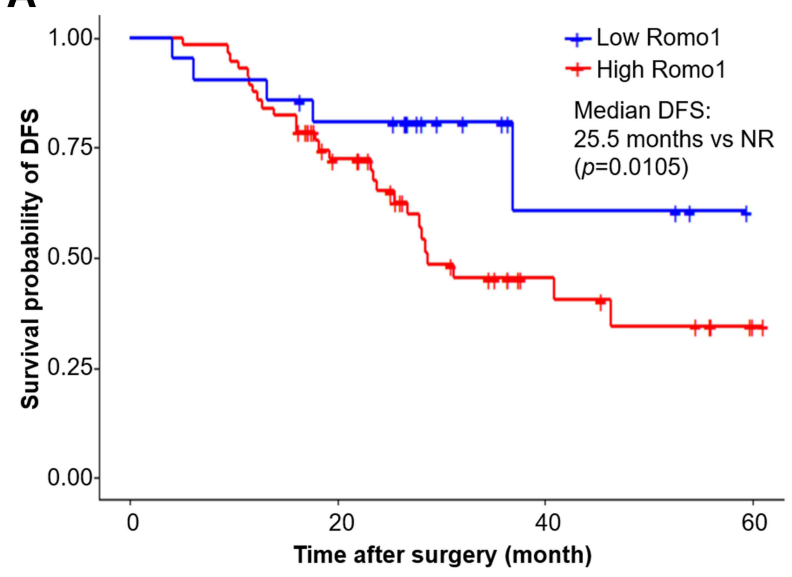

B

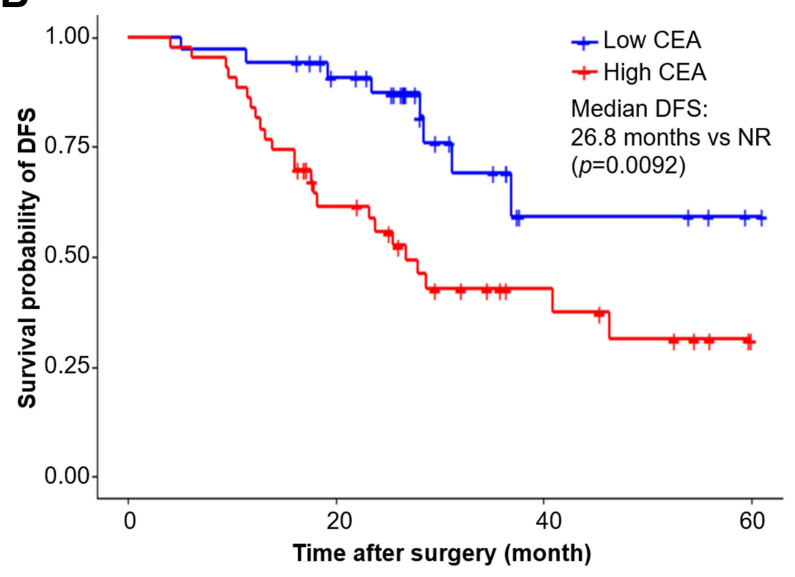

C

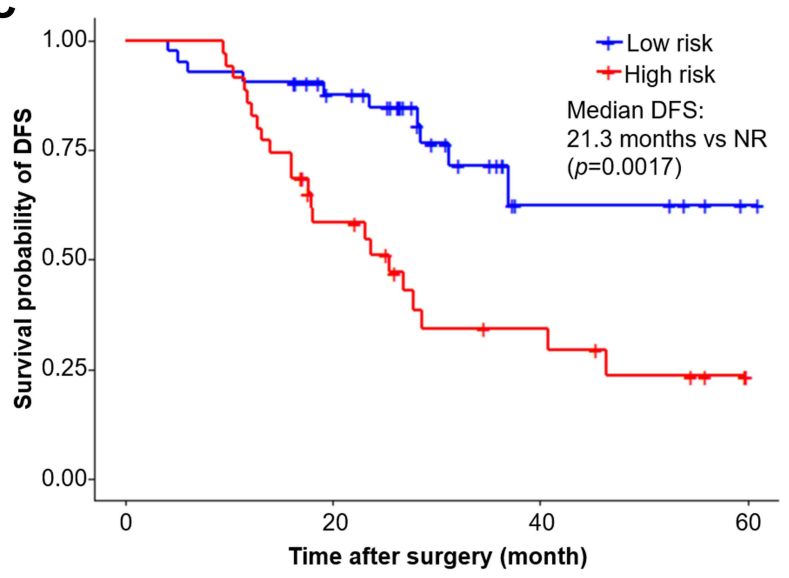

Figure I Kaplan-Meier curves for cumulative disease-free survival according to Romol (A), CEA (B), and the combination of two biomarkers (C). $p$ values were determined by the Log rank test.

Abbreviations: Romol, reactive oxygen species modulator I; CEA, carcinoembryonic antigen; NR, not reached. 
production, and Romol-induced ROS play a significant role in cell proliferation and chemoresistance. ${ }^{12,16,25}$ Furthermore, Romo1 overexpression leads to increased invasive activity of several cancer cells. ${ }^{15,18}$ These findings suggest likely involvement of Romol in cancer progression and clinical outcomes of patients with cancer. Recent clinical studies have demonstrated this perception in various clinical settings for lung cancer. High Romo1 expression in tumor tissue was significantly associated with poor $\mathrm{DFS}(\mathrm{HR}=3.16,95 \% \mathrm{CI}: 1.21-8.22)$ and poor OS $(\mathrm{HR}=3.22$, 95\% CI: 1.02-10.21) in patients with NSCLC who underwent surgical resection ${ }^{20}$ along with poor PFS (HR=2.75, 95\% CI: $1.71-4.44)$ and poor OS $(\mathrm{HR}=3.99$, 95\% CI: 2.36-6.74) in patients with NSCLC who received chemotherapy. ${ }^{19}$ Recent study has reported that Romol overexpression was associated with poor PFS $(\mathrm{HR}=1.87,95 \% \mathrm{CI}: 1.02-4.23)$ and poor $\mathrm{OS}(\mathrm{HR}=2.79$, 95\% CI: 1.13-6.87) in patients treated with definitive radiotherapy. $^{22}$ These consistent data suggest that tissue Romol expression is a promising biomarker for lung cancer in variable clinical settings.

The clinical implications of serum Romol has been scarcely investigated. A previous study demonstrated that serum Romol was significantly higher in patients with lung cancer than in the healthy population or patients with benign lung diseases, suggesting the potential diagnostic value of serum Romo1. ${ }^{26}$ In addition, serum Romo1 level was correlated with tissue Romol expression in lung cancer patients. ${ }^{26}$ Based on these data, we investigated the potential predictive value of serum Romol for patients with surgically resected lung cancer, and successfully demonstrated that high Romol levels were associated with early recurrence. Although serum level of Romol was significantly higher in advanced disease, Romol level was an independent predictive factor in the multivariate analysis after adjusting for confounding factors. This suggests that high Romol level is not only associated with more progressive disease or elevated tumor burden but also reflects more aggressive tumor behavior or metastatic potential independent of tumor stage. Of note, both CEA and Romol levels were independently associated with early recurrence, and the predictive value of Romol was comparable to that of CEA according to HR in the multivariate analysis (2.19 and 2.95 , respectively). In addition, combining these two biomarkers resulted in increased HR (4.11) for DFS, which highlight the possible usefulness of the combination of these two biomarkers for the prediction of recurrence after lung cancer surgery. In the explorative OS analysis, although a significant difference was not observed, a decreasing trend of 4-YSR in the high Romo1 group was observed, similar to that of CEA. The negative results in OS analysis can be attributable to the heterogeneity of therapy after recurrence because different therapies such as chemotherapy, targeted therapy, and immunotherapy were used for appropriate patients. The positive association between Romol and recurrence is in accordance with previous findings on tissue Romol expression in patients with NSCLC, ${ }^{20}$ and thus promising the value of serum Romol as a novel predictor in malignancies. Further large-scaled studies with long term follow-up data are required to confirm the findings of the current study.

The mechanism underlying the relationship between Romo1 level and recurrence or poor survival in patients with cancer is likely attributed to the association of Romol with invasiveness and aggressiveness of cancer cells via modulation of intracellular ROS production. ${ }^{18}$ ROS are involved in cancer progression, including migration, invasion, and angiogenesis through various complex pathways. ${ }^{27}$ Romo1, a mitochondrial membrane protein, is a key regulator of ROS generation, and Romo1-derived ROS are indispensable for cancer cell proliferation. ${ }^{10,12}$ In addition, Romol overexpression has been associated with vascular invasion or lymph node metastasis in patients with $\mathrm{HCC}$ and CRC. ${ }^{17,18}$ In the present study, high serum Romol level was corresponding to advanced stage and poorly differentiated tumors, which are well-known prognostic factors for lung cancer. ${ }^{28}$ Although validation studies are needed, these data highlight that Romol could be critically involved in the aggressive phenotypes of various malignancies.

Interestingly, out data showed that high serum Romo1 level was more relevant to EGFR-wildtype tumor. Upregulated Romo1 expression was consistently observed in a variety of cancer cells, and there was no difference in Romo1 expression according to different histologic subtypes of lung cancer. ${ }^{10,19,20,26}$ The association between Romol expression and EGFR mutation was evaluated and found to be lacking in two previous studies. ${ }^{19,20}$ Unexpectedly, our results revealed different serum Romol levels according to the presence of EGFR mutation. A possible explanation for this finding is based on studies demonstrating that intracellular ROS levels in lung adenocarcinoma cells vary depending on their EGFR mutational status. In one study, baseline ROS levels were significantly higher in EGFR-wildtype (A549) cells than in 
EGFR-mutant (PC 9) cells. $^{29}$ Other studies showed that chronic gefitinib treatment and cigarette smoke extract induced drug resistance by promoting ROS generation in lung cancer cells. ${ }^{29,30}$ The cause of the enhanced ROS level in the EGFR-mutant cells is not clear; however, upregulated Romol could play a certain role in the mechanism. To address this issue, we are now performing an in vitro study on the differential Romol expression in oncogene-addicted lung cancer cells.

Our study has several limitations to be considered. First, this is a relatively small, proof-of-concept study performed in a single institution and the follow-up period is not sufficient. The aim of this study was to provide fundamental data for further large-scaled studies rather than to draw a concrete conclusion. To compensate for the small sample size, we simultaneously measured CEA levels and compared the predictive values of both biomarkers. Even with small sample size and relatively short follow-up period, our data showed possible predictive value of serum Romol levels. Second, we measured only baseline serum Romol levels before surgery and did not analyze changes in its levels during follow-up. Although we successfully showed the clinical applicability of the baseline level, the dynamics before and after surgery could also be valuable in predicting cancer recurrence. Third, we did not explore the value of serum Romo1 level in other histology or clinical situations; therefore, our data cannot be generalized to lung cancer management. To that end, we are currently conducting studies to explore the clinical implications of serum Romo1 level in different histologic subtypes and various treatment settings.

\section{Conclusion}

In conclusion, elevated serum Romo1 levels were significantly associated with early recurrence in patients with lung adenocarcinoma treated with surgical resection. Serum Romo1 levels could be a potential biomarker for predicting recurrence in these patients. Our data provide evidence that measuring Romol level may contribute to improvement in the clinical outcomes by predicting the recurrence risk and selecting high-risk patients who may require adjuvant treatment or other customized approaches. Further investigations are required to determine the usefulness of this biomarker for other malignancies or clinical situations, including targeted therapy and immunotherapy.

\section{Acknowledgments}

The biospecimens and data used in this study were provided by the Biobank of Kyung Hee University Hospital (2020-5). This research was supported by a grant from the Basic Research Program through the National Research Foundation funded by the Ministry of Science and ICT (2019R1F1A1041812) of the Republic of Korea.

\section{Disclosure}

The authors declare that they have no competing interests.

\section{References}

1. Bray F, Ferlay J, Soerjomataram I, Siegel RL, Torre LA, Jemal A. Global cancer statistics 2018: GLOBOCAN estimates of incidence and mortality worldwide for 36 cancers in 185 countries. CA Cancer J Clin. 2018;68(6):394-424.

2. Park CK, Kim SJ. Trends and updated statistics of lung cancer in Korea. Tuberc Respir Dis (Seoul). 2019;82(2):175-177.

3. Park JY, Jang SH. Epidemiology of lung cancer in Korea: recent trends. Tuberc Respir Dis (Seoul). 2016;79(2):58-69.

4. Kim HC, Jung CY, Cho DG, et al. Clinical characteristics and prognostic factors of lung cancer in Korea: a pilot study of data from the Korean nationwide lung cancer registry. Tuberc Respir Dis (Seoul). 2019;82(2):118-125.

5. Garon EB, Hellmann MD, Rizvi NA, et al. Five-year overall survival for patients with advanced non-small-cell lung cancer treated with pembrolizumab: results from the Phase I KEYNOTE-001 study. $J$ Clin Oncol. 2019;37(28):2518-2527.

6. Barlesi F, Gimenez C, Torre JP, et al. Prognostic value of combination of Cyfra 21-1, CEA and NSE in patients with advanced non-small cell lung cancer. Respir Med. 2004;98(4):357-362.

7. Chen F, Wang XY, Han XH, Wang H, Qi J. Diagnostic value of Cyfra21-1, SCC and CEA for differentiation of early-stage NSCLC from benign lung disease. Int $J$ Clin Exp Med. 2015;8 (7):11295-11300.

8. Moro D, Villemain D, Vuillez JP, Delord CA, Brambilla C. CEA, CYFRA21-1 and SCC in non-small cell lung cancer. Lung Cancer. 1995;13(2):169-176.

9. Molina R, Filella X, Auge JM, et al. Tumor markers (CEA, CA 125, CYFRA 21-1, SCC and NSE) in patients with non-small cell lung cancer as an aid in histological diagnosis and prognosis. Comparison with the main clinical and pathological prognostic factors. Tumour Biol. 2003;24(4):209-218.

10. Chung YM, Kim JS, Yoo YD. A novel protein, Romo1, induces ROS production in the mitochondria. Biochem Biophys Res Commun. 2006;347(3):649-655.

11. Lee GY, You DG, Lee HR, Hwang SW, Lee CJ, Yoo YD. Romo1 is a mitochondrial nonselective cation channel with viroporin-like characteristics. J Cell Biol. 2018;217(6):2059-2071.

12. Na AR, Chung YM, Lee SB, Park SH, Lee MS, Yoo YD. A critical role for Romo1-derived ROS in cell proliferation. Biochem Biophys Res Commun. 2008;369(2):672-678.

13. Amini MA, Talebi SS, Karimi J. Reactive oxygen species Modulator 1 (ROMO1), a new potential target for cancer diagnosis and treatment. Chonnam Med J. 2019;55(3):136-143.

14. Chung JS, Lee S, Yoo YD. Constitutive NF-kappaB activation and tumor-growth promotion by Romo1-mediated reactive oxygen species production. Biochem Biophys Res Commun. 2014;450 (4):1656-1661. 
15. Lee S, Park YH, Chung JS, Yoo YD. Romo1 and the NF-kappaB pathway are involved in oxidative stress-induced tumor cell invasion. Int J Oncol. 2015;46(5):2021-2028.

16. Hwang IT, Chung YM, Kim JJ, et al. Drug resistance to 5-FU linked to reactive oxygen species modulator 1. Biochem Biophys Res Commun. 2007;359(2):304-310.

17. Kim HJ, Jo MJ, Kim BR, et al. Reactive oxygen species modulator-1 (Romo1) predicts unfavorable prognosis in colorectal cancer patients. PLoS One. 2017;12(5):e0176834.

18. Chung JS, Park S, Park SH, et al. Overexpression of Romo1 promotes production of reactive oxygen species and invasiveness of hepatic tumor cells. Gastroenterology. 2012;143(4):1084-1094e1087.

19. Lee SH, Choi SI, Lee JS, et al. Reactive Oxygen Species Modulator 1 (Romo1) predicts poor outcomes in advanced non-small cell lung cancer patients treated with platinum-based chemotherapy. Cancer Res Treat. 2017;49(1):141-149.

20. Lee SH, Min JW, Lee JS, et al. Reactive oxygen species modulator 1 (Romo1) overexpression is an independent predictor of poor survival in NSCLC patients who undergo surgical resection. Lung Cancer. 2015;87(1):45-52.

21. Kong M, Sung JY, Lee SH. Reactive Oxygen Species Modulator 1 is associated with poor survival in patients with non-small cell lung cancer after stereotactic fractionated radiosurgery: a retrospective pilot study. Onco Targets Ther. 2020;13:8173-8180.

22. Kong M, Sung JY, Lee SH. Reactive Oxygen Species Modulator 1 as an adverse prognostic marker in Stage III non-small cell lung cancer treated with radiotherapy: a retrospective pilot study. Onco Targets Ther. 2019;12:8263-8273.
23. Goldstraw P, Chansky K, Crowley J, et al. The IASLC Lung Cancer Staging Project: proposals for Revision of the TNM Stage Groupings in the Forthcoming (Eighth) Edition of the TNM classification for lung cancer. J Thorac Oncol. 2016;11(1):39-51.

24. Yoo YA, Kim MJ, Park JK, et al. Mitochondrial ribosomal protein L41 suppresses cell growth in association with p53 and p27Kip1. Mol Cell Biol. 2005;25(15):6603-6616.

25. Shin JA, Chung JS, Cho SH, Kim HJ, Yoo YD. Romo1 expression contributes to oxidative stress-induced death of lung epithelial cells. Biochem Biophys Res Commun. 2013;439(2):315-320.

26. Lee SH, Lee JS, Lee EJ, et al. Serum reactive oxygen species modulator 1 (Romo1) as a potential diagnostic biomarker for non-small cell lung cancer. Lung Cancer. 2014;85(2):175-181.

27. Wu WS. The signaling mechanism of ROS in tumor progression. Cancer Metastasis Rev. 2006;25(4):695-705.

28. Lee SH. Chemotherapy for lung cancer in the era of personalized medicine. Tuberc Respir Dis (Seoul). 2019;82(3):179-189.

29. Zhang L, Li J, Hu J, et al. Cigarette smoke extract induces EGFR-TKI resistance via promoting EGFR signaling pathway and ROS generation in NSCLC cell lines. Lung Cancer. 2017;109:109-116.

30. Okon IS, Coughlan KA, Zhang M, Wang Q, Zou MH. Gefitinibmediated reactive oxygen specie (ROS) instigates mitochondrial dysfunction and drug resistance in lung cancer cells. J Biol Chem. 2015;290(14):9101-9110.

\section{Publish your work in this journal}

OncoTargets and Therapy is an international, peer-reviewed, open access journal focusing on the pathological basis of all cancers, potential targets for therapy and treatment protocols employed to improve the management of cancer patients. The journal also focuses on the impact of management programs and new therapeutic agents and protocols on patient perspectives such as quality of life, adherence and satisfaction. The manuscript management system is completely online and includes a very quick and fair peer-review system, which is all easy to use. Visit http://www.dovepress.com/ testimonials.php to read real quotes from published authors 